\title{
Low luminance visual acuity as a clinical measure and clinical trial outcome measure: A scoping review.
}

Laura J Wood ${ }^{1,2^{*}}$, Jasleen K Jolly1,2, Thomas M W Buckley², Amandeep S Josan ${ }^{1,2}$ Robert E MacLaren.1,2

${ }^{1}$ Nuffield Laboratory of Ophthalmology, Nuffield Department of Clinical, Neurosciences, University of Oxford, Oxford, United Kingdom.

20xford Eye Hospital, Oxford University Hospitals NHS Foundation Trust, Oxford, United Kingdom.

Short Title: Low Luminance Visual Acuity Review

${ }^{*}$ Corresponding Author

Laura Wood

E-mail: enquires@ndcn.ox.ac.uk

Keywords: Low luminance visual acuity, mesopic vision, mesopic visual acuity, low light visual acuity,low luminance deficit. 


\begin{abstract}
Purpose:

The measurement of standard visual acuity (VA) is the most well-known part of any ophthalmic examination to indicate visual function. Despite this, it is insensitive in detecting early disease changes. Therefore, other visual function tests have been developed including low luminance VA (LLVA) and low luminance deficit (LLD). This scoping literature review aims to summarise the current published applications of LLVA and LLD assessments to evaluate their utility as clinical markers and research outcome measures in a variety of ophthalmic conditions.
\end{abstract}

\title{
Recent Findings:
}

Sixty-five peer-reviewed publications were included. LLVA was pioneered for use in geographic atrophy, a subtype of age-related macular degeneration, which remains the mainstay of its clinical application. However, other studies have reported additional useful applications in inherited retinal diseases including rare maculopathies and rod-cone dystrophies. Although there are some variations in testing methodology, use of the standard Early Treatment Diabetic Retinopathy Study (ETDRS) chart with a 2.0 log unit neutral density filter is the most popular approach. The optimal testing luminance is still to be defined.

\section{Summary:}

Overall, LLVA is an earlier clinical marker of change in central retinal function than standard VA. It has been shown to be a risk factor for disease progression and a better indicator of a patient's level of everyday visual function. It is inexpensive and simple to implement using readily available standard ophthalmic equipment. 


\subsection{Introduction}

The measurement of standard visual acuity (VA) is the basis of any visual function examination despite it being insensitive to early disease, or unable to differentiate different disease stages in many retinal conditions..$^{1-4}$ It is well established that vision under low luminance conditions is reduced compared with standard VA.5-8 This has led to the development of the low luminance VA test (LLVA). 9,10

Standard VA measurement is performed under photopic conditions and represents central foveal cone function. Mesopic conditions involve lower light levels, encompassing light intensities from 0.01 to $10 \mathrm{~cd} / \mathrm{m}^{2}$, which is equivalent to moonlight and standard indoor lighting. ${ }^{11}$ It is assumed for mesopic vision that both rods and cones are active; however the exact physiology is unknown. ${ }^{12,13}$

A number of terms describe standard, high contrast, photopic VA; here, this will be simply be referred to as standard VA. Similarly, there are a number of terms describing visual assessment in low light, including mesopic VA and LLVA; we will use the latter description. The low luminance deficit (LLD) is the difference between standard and low luminance VA, and is reported frequently. ${ }^{9,10}$

Several aspects of LLVA assessment have been investigated, including influencing factors and application under different ocular conditions.5, 8, 9, 14-16 Despite this work, there is currently no standardised method of performing the test.1,17 In this scoping review, we aim to analyse the applications of LLVA and summarise the findings, to determine the test merits, optimum methodology and scope for future investigation. 


\subsection{Method}

A literature search was conducted up to 1 April 2020, using MEDLINE and EMBASE. The inclusion criteria allowed any publication that referred to measuring VA in low light conditions. Table 1 details the literature search terms and the screening exclusion criteria applied. After removing duplicates, 498 results (including conference abstracts) were excluded following abstract or full paper screening; 65 peer reviewed publications were subsequently included (Figure 1).

\subsection{Discussion and Critical Review}

\section{$\underline{3.1 \text { Reported uses of low luminance visual acuity in retinal disease }}$}

There has been extensive application of LLVA and LLD in dry age-related macular degeneration (AMD) and geographic atrophy, but only one application in wet AMD. ${ }^{18}$ Seven publications were retrieved investigating the use of LLVA in inherited retinal conditions (Table 2). These results highlight a need for further investigation in other ocular diseases such as diabetic eye disease and specific maculopathies.

\subsection{Low luminance visual acuity Testing Methods \& Validity}

There are differences in the LLVA measurement methods and techniques used to attain mesopic luminance across different studies and research centres (Table 3). Some vary chart light levels, whilst others use neutral density (ND) filters to reduce the luminance level entering the eye. There is also inconsistency in the mesopic luminance level used and period of dark adaptation undertaken. If LLVA is adopted as a routine clinical measure, it is important to establish an optimised and standardised methodology. Here we review the findings from the more popular methodologies to determine the optimum approach. 


\subsubsection{Background Luminance Level}

Lin et al. ${ }^{70}$ investigated LLVA in 40 healthy subjects at luminance levels of 3.0 $\mathrm{cd} / \mathrm{m}^{2}, 0.75 \mathrm{~cd} / \mathrm{m}^{2}$ and $0.38 \mathrm{~cd} / \mathrm{m}^{2}$ using both reduced monitor luminance and ND filters. They found that $0.75 \mathrm{~cd} / \mathrm{m}^{2}$ provided clinically significant and repeatable results. Johnson and Casson also used ND filters to reduce luminance levels to 75 $\mathrm{cd} / \mathrm{m}^{2}, 7.5 \mathrm{~cd} / \mathrm{m}^{2}, 0.75 \mathrm{~cd} / \mathrm{m}^{2}$ and $0.075 \mathrm{~cd} / \mathrm{m}^{2}$, in conjunction with a highresolution monitor. They found that decreasing background luminance was associated with a linear decrease in LLVA. ${ }^{8}$ Similarly, Rabin ${ }^{5}$ reported that increasing luminance from $0.23 \mathrm{~cd} / \mathrm{m}^{2}$ to $116 \mathrm{~cd} / \mathrm{m}^{2}$ generated a three times increase in VA in five healthy subjects. For each doubling of light intensity, there was a corresponding improvement of VA of approximately two letters. Rabin reported that the VA changes between $100-1.0 \mathrm{~cd} / \mathrm{m}^{2}$ remained within normal clinical limits (i.e., around 6/6). VA was only significantly decreased when the background luminance intensity was less than $1.0 \mathrm{~cd} / \mathrm{m}^{2}$. Cocce et al. ${ }^{24}$ reported improved LLVA sensitivity in early and intermediate AMD subjects at a background luminance of $0.5 \mathrm{~cd} / \mathrm{m}^{2}$. Therefore, for significant LLVA results in both healthy and disease groups, the target background luminance should be $<1.0 \mathrm{~cd} / \mathrm{m}^{2}$. Small variations in luminance levels are likely to be clinically insignificant; however, a wide range of luminance levels could vary visual performance. This is a limiting factor of LLVA, which could be improved if a standard target low luminance level was established and the luminance level recorded alongside the LLVA measure.

\subsection{Log Unit Neutral Density Filter}

Five studies from the University of Alabama used an electronic letter chart with a $1.5 \log$ unit ND filter. $27,28,35,67,71$ Four of these studies reported limited discriminatory value for LLVA compared with standard VA. Owsley et al. ${ }^{35}$ 
observed that whilst impaired LLVA was a risk factor for the development of early AMD, LLVA remained stable over the three-year study period, thereby limiting the usefulness as a clinical trial outcome measure. Further, Owsley et al. ${ }^{27}$ also reported no significant difference in LLVA between early AMD subjects and healthy age-matched controls. Similarly, LLD was not sufficiently sensitive to quantify self-reported low luminance difficulties in healthy subjects. ${ }^{67}$ Also, Neely et al. reported LLVA was insensitive to the presence of subretinal drusenoid deposits. ${ }^{28}$ LLVA may have reduced sensitivity compared to standard VA when using the $1.5 \log$ unit ND filter, due to insufficiently dark conditions; this was also suggested by Owsley et al. ${ }^{35}$ However, using the same test set up, Crosson et al. ${ }^{71}$ found both LLVA and rod mediated dark adaption was significantly worse in eyes with pathological features (such as epiretinal membranes and macular telangiectasia type 2) detected with optical coherence tomography, but judged healthy by colour fundus photography.

\subsection{Log Unit Neutral Density Filter Method}

Sunness et al. ${ }^{10}$ utilised the Early Treatment of Diabetic Retinopathy Study (ETDRS) letter chart with a $2.0 \log$ unit ND filter to provide LLVA and LLD values for subjects with geographic atrophy. This is emerging as the standard LLVA approach, since 26 studies (mostly randomised control trials for AMD) have adopted the method. Of these, 81\% (21/26) reported that LLVA or LLD provided useful additional information beyond standard VA. In five studies, LLD has been shown to be a strong predictor of geographic atrophy progression and subsequent VA loss. 10,38,39,41,43 LLD is significantly reduced in patients with macular telangiectasia type 1 , choroideremia and pseudoxanthoma elasticum when compared with healthy controls. ${ }^{44,45,50}$ LLVA has been recommended as a clinical trial outcome measure for dry AMD, geographic atrophy and retinitis pigmentosa. ${ }^{25,37,75}$ In an interventional trial assessing a potential treatment for geographic atrophy, LLVA was significantly reduced in subjects receiving higher 
doses, thus proving to be a useful safety marker of retinal toxicity. ${ }^{38}$ In wet AMD subjects receiving anti-VEGF treatment, LLD showed strong prognostic value, independent of standard VA. A higher proportion of subjects with a small difference between standard VA and LLVA (i.e., small LLD) at screening subsequently experienced significant three-line and six-line standard VA gains compared to those with a larger LLD at screening, who went on to experience larger standard VA declines. ${ }^{18}$ Although these findings were from a large randomised controlled trial (with 1,084 subjects), they are yet to be replicated.

\subsubsection{2.0 Log Unit Neutral Density Filter Luminance Level}

Pondorfer et al. ${ }^{2}$ showed that LLVA differed significantly from standard VA in cases of intermediate AMD, with a $2.0 \log$ ND filter reducing ETDRS chart illumination to $1.5 \mathrm{~cd} / \mathrm{m}^{2}$. The background luminance level is a critical factor in the significance of the results. The average ETDRS chart luminance is $160 \mathrm{~cd} / \mathrm{m}^{2}, 76$ while a $2.0 \log$ ND filter reduces luminance by 100 fold. Hence, with the standard ETDRS chart as the only room luminance source, attaining the previously recommended luminance of less than $1.0 \mathrm{~cd} / \mathrm{m}^{2}$ is unlikely with a $2.0 \log$ unit ND filter, since the achieved low luminance level is likely to be around $1.6 \mathrm{~cd} / \mathrm{m}^{2}$. Given the widespread application and usefulness of this particular approach, this may still be adequate.

Strategies to achieve darker conditions include using chart bulb filters to reduce standard ETDRS luminance to $85 \mathrm{~cd} / \mathrm{m}^{2} 76$ before introducing the $2.0 \log$ ND filter (to create $0.85 \mathrm{~cd} / \mathrm{m}^{2}$ LLVA conditions). Alternatively, using denser ND filters (if available) such as a $2.5 \log$ unit ND filter would reduce luminance by $316 \mathrm{x}$ to 0.5 $\mathrm{cd} / \mathrm{m}^{2}$, or a $2.3 \log$ unit ND filter generating a 200 -fold luminance decrease $(0.8$ $\mathrm{cd} / \mathrm{m}^{2}$ ). These may improve test sensitivity, although the effect of different light levels on the LLVA may only be small in concordance with Rabin et al. ${ }^{5}$ In 
addition, darker conditions may increase floor effects in subjects with more advanced retinal disease.

\subsubsection{Normal Low Luminance Deficit Values}

To obtain a standard LLD for healthy individuals, control data were plotted from six separate studies using the ETDRS chart with a 2.0 log ND filter method (Figure 2). The combined mean LLD was 10 ETDRS letters, with five of the studies showing a 95\% confidence upper limit of 13 ETDRS letters or less. Therefore, this suggests that patients with a LLD of more than 13 ETDRS letters warrant further investigation. However, the combined mean age for the healthy control subjects was 64 years. Hess et al. ${ }^{45}$ reported a mean LLD of $6.1( \pm 1.4)$ ETDRS letters in a younger sample (mean age 52.2, range 23-72). LLVA in healthy individuals declines with age, more than standard VA, thereby resulting in a larger LLD. 14,17,72 This change with age may be due to decreased cone density 77 or lens opacities. Further investigation to provide a more comprehensive normal (healthy) LLD range for younger patients would be of great relevance in the application of LLVA in diabetic retinopathy or inherited retinal conditions where the patients are of working age. However, for now a LLD greater than 13 letters provides a conservative estimate to prompt further investigation.

\subsubsection{Standard versus Computer Testing}

Computer based VA systems use is increasing, as they offer versatile testing options. There are two ways to achieve LLVA with a computerised chart. For example, two studies reported robust and reproducible LLVA results using a computerised testing chart (Innova Systems, www.innovasystemsusa.com/), with the background luminance adjusted to $1.3 \mathrm{~cd} / \mathrm{m}^{2}$ or $0.5 \mathrm{~cd} / \mathrm{m}^{2} .{ }^{15,22}$ Increased sensitivity was found with a $0.5 \mathrm{~cd} / \mathrm{m}^{2}$ background luminance computer chart compared to the standard ETDRS chart with a $2.0 \log$ ND filter. ${ }^{24}$ 
The alternative approach is to maintain the standard monitor illumination settings and apply ND filters to reduce the luminance levels. ${ }^{5,8}$ However, further investigation is required to determine the level of agreement with the standard ETDRS chart in conjunction with a $2.0 \log$ unit ND filter.

\subsubsection{Dark Adaptation}

It is known that sensitivity to low light improves after dark adaptation. ${ }^{11}$ Accordingly, variable periods of dark adaptation have been applied prior to LLVA measures (Table 4); however, only a couple of studies have attempted to assess the duration of dark adaptation on LLVA systematically.5,9 Rabin reported that maximum VA improvement occurred after six minutes of adaptation in healthy subjects. ${ }^{5}$ Conversely, Sunness et al. ${ }^{9}$ concluded that dark adaptation was not required, as 65 subjects with geographic atrophy did not display clinically significant changes following five minutes of adaptation. Furthermore, Hess et al. ${ }^{45}$ reported no link between impaired dark adaptation and LLVA, concluding that LLVA does not represent a surrogate marker for dark adaptation defects. However, it remains uncertain whether dark adaptation influences LLVA variability, either in healthy controls or cases of retinal disease where the rate of dark adaptation may be impaired. It is unclear whether a longer LLVA test duration, and hence more dark adaptation time, could bias results. Since the left eye is generally tested after the right eye, it may be at an advantage as it has a longer time to dark-adapt behind the occluder, thereby increasing retinal sensitivity before the LLVA measurement. Further investigation is required to determine this.

\subsubsection{Test Retest Repeatability}


Several studies have reported LLVA test-retest repeatability values between \pm 0.1 to $0.13 \operatorname{LogMAR}$ ( 5 to 6.5 ETDRS letters) in both healthy subjects and those with AMD, 1,22,23,68 despite adopting different methodologies to generate low luminance conditions (Table 3). This level of test-retest repeatability is comparable with the standard VA repeatability of $\pm 0.15 \log$ MAR. ${ }^{78}$

\subsection{Links to visual function}

LLVA is hypothesised to be a better marker of everyday visual function under low light conditions than standard VA. And yet studies involving visually healthy, older age groups reported that neither LLVA nor LLD were associated with significant changes in quality of life measures or disability questionnaires.435,72 This contrasts with AMD studies where quality of life measures correlated significantly with LLVA and LLD in all stages of the disease.16,19-21

\subsubsection{Driving}

The Salisbury Eye Evaluation Study investigated whether LLVA (luminance level $5.2 \mathrm{~cd} / \mathrm{m}^{2}$ ) is a useful marker of nighttime driving ability, but found that LLVA was not a significant predictor of car crash risk. ${ }^{62}$ Sivak and Olson ${ }^{63}$ reported that the nighttime driving performance of six older and six younger drivers was comparable when their LLVA scores were matched. Despite the small sample size, these authors recommended LLVA as a more relevant evaluation of nighttime driving than standard VA, and concluded that visual deficits, rather than difficulties with information processing, were responsible for poor driving performance. The differences between these two studies likely stems from the different luminance levels adopted. Two additional investigations reported that LLVA was a better predictor of driving visual performance than standard VA, and therefore should be included in driving visual assessments. ${ }^{14,17}$ Before any formal 
driving standard recommendations can be made, the levels of LLVA deemed safe for driving must be determined.

\subsection{Low Luminance Visual Acuity Physiological Functional Mechanisms}

LLVA is widely considered to be reflective of foveal cone function, due to a high correlation with standard VA and cone contrast testing. ${ }^{18,24,79}$ Although LLVA is less affected by crowding effects, suggesting different spatial processing to standard VA. ${ }^{1}$ However, the exact functional mechanism behind LLVA remains unclear.

Owsley et al. ${ }^{35}$ discussed three possible physiological functional mechanisms related to LLVA. Firstly, reduced LLVA may reflect compromised cone sampling density in the central fovea and impaired cone mediated resolution. Advances in adaptive optics could investigate this proposal. ${ }^{77}$ Secondly, rod photoreceptors may contribute to LLVA via rod-cone coupling in the parafoveal region. Rod degeneration may result in reduced central foveal sensitivity. However, a recent study suggested no link to rod function, as there was no significant correlation between AdaptDx (www.maculogix.com) scotopic rod function and LLVA in patients with retinitis pigmentosa. ${ }^{47}$ The same study reported a significant correlation between perifoveal scotopic cone function and LLVA, supporting the notion that LLVA reflects foveal cone function. The third proposed mechanism is that LLVA depends on cone-to-cone circuits, via horizontal and amacrine cells in the plexiform layers. ${ }^{13,35}$ It is possible that LLVA is a reflection of multiple mechanisms, including cone sampling density and cone-cone coupling.

An investigation using scanning laser ophthalmoscopy analysed fixation in a patient with progressive maculopathy noted that the preferred retinal locus switched consistently and reproducibly from the fovea in photopic conditions to a 
specific, perifoveal location under mesopic conditions. ${ }^{80}$ The mechanism for this switch was unknown; however, it is possible that the preferred retinal locus, in low light, is driven to larger areas of preserved retinal sensitivity. This suggests that LLVA is dependent on a critical area or volume of preserved retinal sensitivity, and therefore a minimum 'cone-circuit'.

Pelli-Robson contrast sensitivity and LLVA are highly correlated in healthy individuals, suggesting that both tests reflect similar retinal function.1,17,47 As they assess intermediate spatial frequencies, they may represent functional spatial neural summation. ${ }^{11}$ However, the different light level arrangements for each test may reflect different functional mechanisms. ${ }^{50}$

It remains unclear whether LLVA is a reflection of central foveal function, as suggested by Sunness et al $^{9}$ or is influenced by parafoveal input. Studies involving parafoveal pathology with a preserved central fovea (e.g., macular telangiectasia type 1, central serous retinopathy and non-foveal geographic atrophy), have reported significantly different LLVA results compared to standard VA.43,44,51,81 In those cases with pathology affecting the central fovea, LLVA was less useful,22 presumably because standard VA was also impaired. For this reason, LLVA and LLD results should not be interpreted in isolation but in unison with standard VA to ensure a comprehensive understanding of central retinal function. Overall, LLVA appears to be a more sensitive and earlier clinical marker of central retinal sensitivity in the presence of good standard VA. 41

\subsection{Limitations}

Efficacy comparisons have been challenging due to the variety of methodologies employed by different study groups (Table 3). Nine investigations did not provide sufficient LLVA methodology details to replicate their study $36,37,42,49,53,58,61,63,65$. 
Even where detailed test methodology was reported, the luminance levels used appeared to be missing. In addition, it is challenging to quantify how useful the application of LLVA is in different scenarios as the sensitivity and specificity of the LLVA test as a screening tool has not been reported.

Where LLVA was used within a natural history of disease study or a randomised control trial, these investigations tended to include large subject numbers.

However, most studies delivering useful insights into the impact of luminance levels on VA included only small participant numbers.5,8 Similarly, in those studying rare diseases, large sample sizes are not feasible, but results can still be valuable. ${ }^{44,45,47,50}$

Finally, many of the studies categorised subjects via fundus image characteristics, such as the Age-Related Eye Disease Studies (AREDS) classification of AMD, 82 as opposed to using newer technologies such as optical coherence tomography to categorise disease subgroups. Despite the success of the AREDS classification systems, ${ }^{83}$ those with reticular pseudodrusen can be difficult to define accurately and may be misclassified. ${ }^{84}$ Similarly, the AREDS system does not take into account different high risk AMD genotypes. ${ }^{85}$ These two limitations could increase variability in the LLVA functions within categorised disease stages.

\subsection{Conclusion}

In summary: 1. The ETDRS chart with a $2.0 \log$ unit ND filter is the most commonly used LLVA testing methodology. 2. Further investigation to establish the recommended target luminance level is required. 3. The mesopic luminance level used should be recorded with the LLVA score to aid consistency and reduce variability. 4. LLVA is likely a marker of foveal and parafoveal cone function in low light. 5. Low LLVA is a risk factor for disease progression in geographic atrophy, but further investigation is required for other ophthalmic diseases. 6 . 
Patients with a LLD above 13 ETDRS letters (0.14 LogMAR) should warrant further clinical investigation. 7. LLVA is a complementary marker to standard VA, indicative of central retinal sensitivity, and should be used in reference to standard VA. Scope for further investigation includes: validating LLVA utility in other retinal conditions, identifying a normal LLD upper limit for younger individuals and standardising computerised testing set ups, and working to understand influencing LLVA variability factors such as dark adaptation or testing at lower light levels. These investigations will help to standardise the test and encourage adoption into clinical practice.

While microperimetry and other visual function tests are potentially more sensitive than LLVA, ${ }^{20,22,33,34,44}$ they require extra equipment, resources and longer test durations. LLVA has the advantage of being inexpensive, repeatable, utilises basic ophthalmic equipment and is simple to conduct.10 It also enables earlier detection of retinal disease changes. The review demonstrates the scope to optimise test methodology for future clinical applications. We believe LLVA should be implemented beyond clinical trials into standard ophthalmological and optometric care.

\section{Disclosure}

The authors report no conflicts of interest and have no proprietary interest in any of the materials mentioned in this article.

\section{Acknowledgements}


The review was funded by a preparatory fellowship from the National Institute for Health Research (NIHR) Oxford Biomedical Research Centre. The views expressed are those of the authors and not necessarily those of the NIHR or the Department of Health and Social Care.

We would like to thank Neil Thurley, subject librarian at the Cairns Library, University of Oxford, for support with constructing the literature search. We would also like to thank Dr Tjebo Heeren, Dr Simone Muller, Professor Sobha Sivaprasad, Manjot Grewal and Associate Professor Chi Luu, as well as the other referenced authors, for the data to enable composition of Table 3. 


\section{References (Numerical)}

1. Pluhacek F, Siderov J. Mesopic visual acuity is less crowded. Graefe's archive for clinical and experimental ophthalmology. 2018;256(9):1739-46.

2. Pondorfer SG, Heinemann M, Wintergerst MWM, Pfau M, Strömer AL, Holz FG, et al. Detecting vision loss in intermediate age-related macular degeneration: A comparison of visual function tests. PLOS ONE. 2020;15(4):e0231748.

3. Jolly JK, Xue K, Edwards TL, Groppe M, MacLaren RE. Characterizing the Natural History of Visual Function in Choroideremia Using Microperimetry and Multimodal Retinal Imaging. Investigative Ophthalmology \& Visual Science. 2017;58(12):5575-83.

4. Rubin GS, Bandeen-Roche K, Huang GH, Munoz B, Schein OD, Fried LP, et al. The association of multiple visual impairments with self-reported visual disability: SEE project. Investigative ophthalmology \& visual science. 2001;42(1):64-72.

5. Rabin J. Luminance effects on visual acuity and small letter contrast sensitivity. Optometry and vision science : official publication of the American Academy of Optometry. 1994;71(11):685-8.

6. Shlaer S. The Relation Between Visual Acuity and Illumination. J Gen Physiol. 1937;21(2):165-88.

7. Sheedy JE, Bailey IL, Raasch TW. Visual acuity and chart luminance. Am J Optom Physiol Opt. 1984;61(9):595-600.

8. Johnson CA, Casson EJ. Effects of luminance, contrast, and blur on visual acuity. Optometry and vision science. 1995;72(12):864-9.

9. Sunness JS, Rubin GS, Applegate CA, Bressler NM, Marsh MJ, Hawkins BS, et al. Visual function abnormalities and prognosis in eyes with age-related geographic atrophy of the macula and good visual acuity. Ophthalmology. 1997;104(10):1677-91.

10. Sunness JS, Rubin GS, Broman A, Applegate CA, Bressler NM, Hawkins BS. Low luminance visual dysfunction as a predictor of subsequent visual acuity loss from geographic atrophy in age-related macular degeneration. Ophthalmology. 2008;115(9):1480-2.

11. SH S. Visual Perception A Clinical Orientation. 4th Edition ed. New York: McGraw Hill Medical; 2010.

12. Stockman A, Sharpe LT. Into the twilight zone: the complexities of mesopic vision and luminous efficiency. Ophthalmic Physiol Opt. 2006;26(3):225-39.

13. Zele AJ, Cao D. Vision under mesopic and scotopic illumination. Frontiers in psychology. 2015;5:1594.

14. Sturgis SP, Osgood DJ. Effects of glare and background luminance on visual acuity and contrast sensitivity: Implications for driver night vision testing. Human Factors. 1982;24(3):347-60.

15. Puell MC, Perez-Carrasco MJ, Palomo-Alvarez C, Antona B, Barrio A. Relationship between halo size and forward light scatter. The British journal of ophthalmology. 2014;98(10):1389-92. 
16. Thompson AC, Luhmann UFO, Stinnett SS, Vajzovic L, Horne A, Toth CA, et al. Association of low luminance questionnaire with objective functional measures in early and intermediate age-related macular degeneration. Investigative Ophthalmology and Visual Science. 2018;59(1):289-97.

17. Wood JM, Owens DA. Standard measures of visual acuity do not predict drivers' recognition performance under day or night conditions. Optometry and vision science. 2005;82(8):698-705.

18. Frenkel REP, Shapiro H, Stoilov I. Predicting vision gains with anti-VEGF therapy in neovascular age-related macular degeneration patients by using lowluminance vision. The British journal of ophthalmology. 2016;100(8):1052-7. 19. Wu Z, Guymer RH, Finger RP. Low luminance deficit and night vision symptoms in intermediate age-related macular degeneration. The British journal of ophthalmology. 2016;100(3):395-8.

20. Pondorfer SG, Terheyden JH, Heinemann M, Wintergerst MWM, Holz FG, Finger RP. Association of Vision-related Quality of Life with Visual Function in Age-Related Macular Degeneration. Scientific reports. 2019;9(1):15326.

21. McGuinness MB, Finger RP, Wu Z, Luu CD, Chen FK, Arnold JJ, et al. Properties of the impact of vision impairment and night vision questionnaires among people with intermediate age-related macular degeneration. Translational Vision Science and Technology. 2019;8(5):3.

22. Wu Z, Ayton LN, Guymer RH, Luu CD. Low-luminance visual acuity and microperimetry in age-related macular degeneration. Ophthalmology. 2014;121(8):1612-9.

23. Chandramohan A, Stinnett SS, Petrowski JT, Schuman SG, Toth CA, Cousins $\mathrm{SW}$, et al. Visual function measures in early and intermediate age-related macular degeneration. Retina. 2016;36(5):1021-31.

24. Cocce KJ, Stinnett SS, Luhmann UFO, Vajzovic L, Horne A, Schuman SG, et al. Visual Function Metrics in Early and Intermediate Dry Age-related Macular Degeneration for Use as Clinical Trial Endpoints. American journal of ophthalmology. 2018;189:127-38.

25. Finger RP, Schmitz-Valckenberg S, Schmid M, Rubin GS, Dunbar H, Tufail A, et al. MACUSTAR: Development and Clinical Validation of Functional, Structural, and Patient-Reported Endpoints in Intermediate Age-Related Macular Degeneration. Ophthalmologica Journal international d'ophtalmologie International journal of ophthalmology Zeitschrift fur Augenheilkunde. 2019;241(2):61-72.

26. Heinemann M, Welker SG, Li JQ, Wintergerst MWM, Turski GN, Turski CA, et al. Impact of visual impairment on physical activity in early and late age-related macular degeneration. PloS one. 2019;14(10):e0222045.

27. Owsley C, Huisingh C, Clark ME, Jackson GR, McGwin G, Jr. Comparison of Visual Function in Older Eyes in the Earliest Stages of Age-related Macular Degeneration to Those in Normal Macular Health. Current eye research. 2016;41(2):266-72.

28. Neely D, Zarubina AV, Clark ME, Huisingh CE, Jackson GR, Zhang Y, et al. 
Association Between Visual Function and Subretinal Drusenoid Desposits in Normal and Early Age-Related Macular Degeneration Eyes. Retina. 2017;37(7):1329-36.

29. Puell MC, Barrio AR, Palomo-Alvarez C, Gomez-Sanz FJ, Clement-Corral A, Perez-Carrasco MJ. Impaired mesopic visual acuity in eyes with early age-related macular degeneration. Investigative ophthalmology \& visual science. 2012;53(11):7310-4.

30. Ou WC, Denlar RA, Csaky KG. The Relationship Between Central Drusen Volume and Low-Luminance Deficit in Age-Related Macular Degeneration. Translational Vision Science \& Technology. 2020;9(4):10.

31. Pondorfer SG, Wintergerst MWM, Gorgi Zadeh S, Schultz T, Heinemann M, Holz FG, et al. Association of Visual Function Measures with Drusen Volume in Early Stages of Age-Related Macular Degeneration. Investigative Ophthalmology \& Visual Science. 2020;61(3):55.

32. Grewal MK, Sivapathasuntharam C, Chandra S, Gurudas S, Chong V, Bird A, et al. A Pilot Study Evaluating the Effects of $670 \mathrm{~nm}$ Photobiomodulation in Healthy Ageing and Age-Related Macular Degeneration. J Clin Med. 2020;9(4):1001.

33. Wu Z, Luu CD, Hodgson LAB, Caruso E, Brassington KH, Tindill N, et al. Secondary and Exploratory Outcomes of the Subthreshold Nanosecond Laser Intervention Randomized Trial in Age-Related Macular Degeneration: A LEAD Study Report. Ophthalmology Retina. 2019;3(12):1026-34.

34. Hsu ST, Thompson AC, Stinnett SS, Luhmann UFO, Vajzovic L, Horne A, et al. Longitudinal Study of Visual Function in Dry Age-Related Macular Degeneration at 12 Months. Ophthalmology Retina. 2019;3(8):637-48.

35. Owsley C, Clark ME, Huisingh CE, Curcio CA, McGwin G. Visual function in older eyes in normal macular health: Association with incident early age-related macular degeneration 3 years later. Investigative Ophthalmology and Visual Science. 2016;57(4):1782-9.

36. Liao DS, Grossi FV, El Mehdi D, Gerber MR, Brown DM, Heier JS, et al. Complement C3 Inhibitor Pegcetacoplan for Geographic Atrophy Secondary to Age-Related Macular Degeneration: A Randomized Phase 2 Trial. Ophthalmology. 2020; 127: 186-195.

37. Krezel AK, Hogg R, Lohfeld L, Chakravarthy U, Azuara-Blanco A. Core outcomes for geographic atrophy trials. The British journal of ophthalmology. 2020; 104: 1196-1202.

38. Rosenfeld PJ, Dugel PU, Holz FG, Heier JS, Pearlman JA, Novack RL, et al. Emixustat Hydrochloride for Geographic Atrophy Secondary to Age-Related Macular Degeneration: A Randomized Clinical Trial. Ophthalmology. 2018;125(10):1556-67.

39. Rosenfeld PJ, Berger B, Reichel E, Danis RP, Gress A, Ye L, et al. A Randomized Phase 2 Study of an Anti-Amyloid beta Monoclonal Antibody in Geographic Atrophy Secondary to Age-Related Macular Degeneration. Ophthalmology Retina. 2018;2(10):1028-40. 
40. Heier JS, Pieramici D, Chakravarthy U, Patel SS, Gupta S, Lotery A, et al. Visual Function Decline Resulting from Geographic Atrophy: Results from the Chroma and Spectri Phase 3 Trials. Ophthalmology Retina. 2020; 4: 673-688. 41. Yehoshua Z, Alexandre De Amorim Garcia Filho C, Nunes RP, Gregori G, Penha FM, Moshfeghi AA, et al. Systemic complement inhibition with eculizumab for geographic atrophy in age-related macular degeneration: The COMPLETE study. Ophthalmology. 2014;121(3):693-701.

42. Fleckenstein M, Mitchell P, Freund KB, Sadda S, Holz FG, Brittain C, et al. The Progression of Geographic Atrophy Secondary to Age-Related Macular Degeneration. Ophthalmology. 2018;125(3):369-90.

43. Holekamp N, Wykoff CC, Schmitz-Valckenberg S, Monés J, Souied EH, Lin H, et al. Natural History of Geographic Atrophy Secondary to Age-Related Macular Degeneration: Results from the Prospective Proxima A and B Clinical Trials. Ophthalmology. 2020; 127: 769-783.

44. Muller S, Heeren TFC, Bonelli R, Fruttiger M, Charbel Issa P, Egan CA, et al. Contrast sensitivity and visual acuity under low light conditions in macular telangiectasia type 2. The British journal of ophthalmology. 2019;103(3):398403.

45. Hess K, Gliem M, Birtel J, Muller P, Hendig D, Andrews C, et al. Impaired Dark Adaptation Associated with a diseased Bruch Membrane in Pseudoxanthoma Elasticum. Retina. 2020; 40: 1988-1995.

46. Pilotto E, Leonardi F, Deganello D, Convento E, Midena E, Frizziero L. Morphofunctional Evaluation of Macular-Foveal Capiliaries: A Comparative Optical Coherence Tomography Angiography and Microperimetry Study.. 2020; 40: 1279-1285.

47. Bittner AK, Ferraz MC. Reliability of Mesopic Measures of Visual Acuity and Contrast Sensitivity and Their Correlation with Rod and Cone Function in Retinitis Pigmentosa. Ophthalmic Res. 2020;63(2):133-40.

48. Menghini M, Cehajic-Kapetanovic J, MacLaren RE. Monitoring progression of retinitis pigmentosa: current recommendations and recent advances. Expert opinion on orphan drugs. 2020;8(2-3):67-78.

49. Taylor SP. Use of light intensifiers in improving acuity in retinitis pigmentosa. The British journal of physiological optics. 1979;33(2):29-32.

50. Wood LJ, Jolly JK, Andrews CD, Wilson IR, Hickey D, Cehajic-Kapetanovic J, et al. Low-contrast visual acuity versus low-luminance visual acuity in choroideremia. Clinical and Experimental Optometry. 2020;n/a(n/a).

51. Fujita K, Shinoda K, Matsumoto CS, Imamura Y, Mizutani Y, Tanaka E, et al. Low luminance visual acuity in patients with central serous chorioretinopathy. Clinical \& experimental optometry. 2013;96(1):100-5.

52. Willcox A, Culliford L, Ellis L, Rogers CA, Cree A, Chakravarthy U, et al. Clinical efficacy of eplerenone versus placebo for central serous chorioretinopathy: study protocol for the VICI randomised controlled trial. Eye (London, England). 2019;33(2):295-303. 
53. Wu ZZ, Ma P, Luo Q, Chen B. Comparison of the visual acuity after implantation of Acrysof and Acryof IQ intraocular lens. International Journal of Ophthalmology. 2008;8(10):2023-5.

54. Carballo J, Puell MC, Cuina R, Vazquez JM, Benitez-del-Castillo JM. Changes in visual function under mesopic and photopic conditions after intrastromal corneal ring segment implantation for different stages of keratoconus. Journal of cataract and refractive surgery. 2013;39(3):393-402.

55. Sanders E, Wagner H, Reich LN. Visual acuity and "balanced progressive" simultaneous vision multifocal contact lenses. Eye \& contact lens. 2008;34(5):293-6.

56. Guillon M, Maissa C, Cooper P, Girard-Claudon K, Poling TR. Visual performance of a multi-zone bifocal and a progressive multifocal contact lens. The CLAO journal : official publication of the Contact Lens Association of Ophthalmologists, Inc. 2002;28(2):88-93.

57. Johnson KL, Carney LG, Mountford JA, Collins MJ, Cluff S, Collins PK. Visual performance after overnight orthokeratology. Contact lens \& anterior eye : the journal of the British Contact Lens Association. 2007;30(1):29-36.

58. Higgins KE. Monocular performance of functional amblyopes: another look at the scotoma hypothesis. American journal of optometry and physiological optics. 1978;55(3):172-82.

59. von Noorden GK, Burian HM. Visual Acuity in Normal and Amblyopic Patients Under Reduced Illumination: I. Behavior of Visual Acuity With and Without Neutral Density Filter. AMA Archives of Ophthalmology. 1959;61(4):5335.

60. Jafarzadehpur E, Yarigholi MR. Comparison of visual acuity in reduced lumination and facility of ocular accommodation in table tennis champions and non- players. Journal of sports science \& medicine. 2004;3(1):44-8.

61. Melcher MH, Lund DR. Sports vision and the high school student athlete. Journal of the American Optometric Association. 1992;63(7):466-74.

62. Rubin GS, Ng ESW, Bandeen-Roche K, Keyl PM, Freeman EE, West SK. A prospective, population-based study of the role of visual impairment in motor vehicle crashes among older drivers: the SEE study. Investigative ophthalmology \& visual science. 2007;48(4):1483-91.

63. Sivak M, Olson PL. Nighttime legibility of traffic signs: Conditions eliminating the effects of driver age and disability glare. Accident Analysis and Prevention. 1982;14(2):87-93.

64. Lenton L. Visual performance in a flight simulator: multifocal intraocular lenses in pilots. BMJ open ophthalmology. 2018;3(1):e000139.

65. Vecchi D, Morgagni F, Guadagno AG, Lucertini M. Visual function at altitude under night vision assisted conditions. Aviation, space, and environmental medicine. 2014;85(1):60-5.

66. Jacobs RJ, Hendicott PL, Murphy B, Poppelwell D, Turner PJ. Visual performance requirements for post-PRK police recruits. Clinical \& experimental optometry. 1998;81(4):163-73. 
67. Owsley C, McGwin G, Jr. Vision-targeted health related quality of life in older adults: patient-reported visibility problems in low luminance activities are more likely to decline than daytime activities. BMC ophthalmology. 2016;16:92. 68. Barrio A, Antona B, Puell MC. Repeatability of mesopic visual acuity measurements using high- and low-contrast ETDRS letter charts. Graefe's archive for clinical and experimental ophthalmology = Albrecht von Graefes Archiv fur klinische und experimentelle Ophthalmologie. 2015;253(5):791-5.

69. Applegate RA, Marsack JD, Thibos LN. Metrics of retinal image quality predict visual performance in eyes with $20 / 17$ or better visual acuity. Optometry and vision science. 2006;83(9):635-40.

70. Lin RJ, Ng JS, Nguyen AL. Determinants and standardization of mesopic visual acuity. Optometry and vision science. 2015;92(5):559-65.

71. Crosson JN, Swain TA, Clark ME, Huisingh CE, McGwin G, Jr., Owsley C, et al. Retinal Pathologic Features on OCT among Eyes of Older Adults Judged Healthy by Color Fundus Photography. Ophthalmology Retina. 2019;3(8):670-80.

72. Puell MC, Perez-Carrasco MJ, Palomo Alvarez C. Macular Thickness and Mesopic Visual Acuity in Healthy Older Subjects. Current eye research. 2019;44(1):82-8.

73. Lin TP, Rigby H, Adler JS, Hentz JG, Balcer LJ, Galetta SL, et al. Abnormal visual contrast acuity in Parkinson's disease. J Parkinsons Dis. 2015;5(1):125-30. 74. Thompson AC, Stinnett S, Luhmann UFO, Oza V, Vajzovic L, Horne A, et al. Longitudinal progression of visual function metrics in early and intermediate agerelated macular degeneration. Investigative Ophthalmology and Visual Science. 2018;59(9).

75. Menghini M, Birch DG, Boon C, Duncan JL, Fischer MD, Holz FG, et al. Natural History of the Progression of RPGR-Associated X-Linked Retinitis Pigmentosa (XOLARIS) Study: Cross-Sectional Analysis of Baseline Characteristics. Investigative Ophthalmology and Visual Science. 2019;60(9). 76. ETDRS Illuminator Cabinet New Lamp Filter Tube - Precision Vision 2011 [Available from: https://www.precision-vision.com/etdrs-illuminator-cabinetnew-lamp-filter-tube-precision-vision/.

77. Legras R, Gaudric A, Woog K. Distribution of cone density, spacing and arrangement in adult healthy retinas with adaptive optics flood illumination. PloS one. 2018;13(1):e0191141-e.

78. Siderov J, Tiu AL. Variability of measurements of visual acuity in a large eye clinic. Acta Ophthalmol Scand. 1999;77(6):673-6.

79. Puell M, Hurtado-Cena FJ, Ciercoles-Rodriguez M, Garcia-Benitez N, GomezPalacios V, Alia-Gonzalez C, et al. Relationship between macular thickness and mesopic visual acuity in early to intermediate age-related macular degeneration subjects. Acta Ophthalmologica. 2018;96(Supplement 261):101.

80. Lei H, Schuchard RA. Using two preferred retinal loci for different lighting conditions in patients with central scotomas. Investigative Ophthalmology \& Visual Science. 1997;38(9):1812-8. 
81. Wu Z, Ayton LN, Luu CD, Guymer RH. Longitudinal changes in microperimetry and low luminance visual acuity in age-related macular degeneration. JAMA ophthalmology. 2015;133(4):442-8.

82. The Age-Related Eye Disease Study system for classifying age-related macular degeneration from stereoscopic color fundus photographs: the AgeRelated Eye Disease Study Report Number 6. Am J Ophthalmol. 2001;132(5):66883. Thee EF, Meester-Smoor MA, Luttikhuizen DT, Colijn JM, Enthoven CA, Haarman AEG, et al. Performance of Classification Systems for Age-Related Macular Degeneration in the Rotterdam Study. Translational Vision Science \& Technology. 2020;9(2):26

84. Wu Z, Ayton LN, Luu CD, Baird PN, Guymer RH. Reticular Pseudodrusen in Intermediate Age-Related Macular Degeneration: Prevalence, Detection, Clinical, Environmental, and Genetic Associations. Invest Ophthalmol Vis Sci. 2016;57(3):1310-6.

85. Domalpally A, Agrón E, Pak JW, Keenan TD, Ferris FL, 3rd, Clemons TE, et al. Prevalence, Risk, and Genetic Association of Reticular Pseudodrusen in Agerelated Macular Degeneration: Age-Related Eye Disease Study 2 Report 21. Ophthalmology. 2019;126(12):1659-66.

Legends

Table 1. The literature search terms and screening exclusion criteria used to conduct the search. 
Table 2. Lists the reported applications of low luminance visual measures by disease subject group and study topics. Patient reported outcome measure (PROM). Age related macular degeneration (AMD).

Table 3. Low luminance visual acuity testing methods described in literature. Neutral density: (ND).

Table 4. Lists the dark adaptation times used in different studies measuring low luminance visual acuity.

Figure 1. The literature search, screening process and results.

Figure 2. Forest plot for low luminance difference (LLD) across six studies (130 subjects) with healthy controls using the ETDRS chart and a 2.0 log unit neutral density (ND) filter to measure low luminance visual acuity (LLVA). The grey dashed line denotes the upper LLD normal limit. 